\section{Arbeitsstoff-Toleranzwert, biologischer}

C. Vidal ${ }^{1}$ und W.-R. Külpmann ${ }^{2}$

${ }^{1}$ Landeskriminalamt Niedersachsen, Dezernat 53 „Chemie“, Hannover, Deutschland

${ }^{2}$ Hannover, Deutschland

Synonym(e) BAT-Wert; Biologische Arbeitsstoff-Toleranz

Englischer Begriff biological tolerance value

Definition Der BAT-Wert ist die beim Menschen höchst zulässige Quantität eines Arbeitsstoffes bzw. -metaboliten oder die dadurch ausgelöste Abweichung eines biologischen Indikators von seiner Norm, die nach dem gegenwärtigen Stand der wissenschaftlichen Kenntnis im Allgemeinen die Gesundheit der Beschäftigten auch dann nicht beeinträchtigt, wenn sie durch Einflüsse des Arbeitsplatzes regelhaft erzielt wird.

Beschreibung Seit dem Jahr 2010 besteht mit dem Inkraftreten der neuen Gefahrstoffverordnung ein neues Grenzwertkonzept. Diese Verordnung kennt nur noch gesundheitsbasierte Grenzwerte, genannt Arbeitsplatzgrenzwert (AGW) und biologischer Grenzwert (BGW). Die alten Bezeichnungen MAK-Wert ( $\triangleright$ Arbeitsplatzkonzentration, maximale) und BAT-Wert können und sollen jedoch bis zur vollständigen Umsetzung der Verordnung als Richt- und Orientierungsgrößen weiter verwendet werden.

\section{Literatur}

DFG (2016) MAK- und BAT-Werte-Liste 2016. Wiley-VCH, Weinheim Die Verordnung zum Schutz vor gefährlichen Stoffen (Gefahrstoffverordnung - GefStoffV) (2010) Bundesgesetzblatt I, S 1643 